\title{
A model for Manufacturing Large Parts with WAAM Technology
}

\author{
Hoang Thanh $\mathrm{VO}^{\mathrm{a}, 1}$, Christelle GRANDVALLET ${ }^{\mathrm{a}}$ and Frédéric VIGNAT \\ Univ. Grenoble Alpes, CNRS, Grenoble INP, G-SCOP, 46, avenue Félix Viallet, F- \\ 38000 Grenoble, France
}

\begin{abstract}
Wire Arc Additive Manufacturing (WAAM) is a metallic additive manufacturing process based on the fusion of metallic wire using an electric arc as a heat source. The challenge associated with WAAM is heat management and understanding bead geometry. The printing process involves high temperatures, which results in the build-up of residual stresses can often cause deformations in a component. All of the process variables, such as torch speed (TS), wire feed speed (WFS), idle time, combine to produce the geometry of the deposit bead that results in the desired component shape. So, determining a method for choosing a good combined parameter process is very important to obtain a high-quality part. This article presents a study on how to use the WAAM process to produce a complexity part of aluminium alloys. The step of the determination process parameter is concentrated to develop in this study. An experimental design is determined to study the influence between the process parameters, for example, WFS, TS, high layer, length of bead. Different samples are made using the Yaskawa robot, using the classic CMT (Cold Metal Transfer) as a manufacturing method, using zigzag filling as a manufacturing strategy with the same WFS and same idle times and different TS, different bead lengths. A new manufacturing method using the zigzag filling strategy is proposed by adding an important step in determining the process parameters. The results indicate that the length of the bead has a significant impact on another parameter of the process.
\end{abstract}

Keywords. Wire arc additive manufacturing, filling strategy, cold metal transfer.

\section{Introduction}

\subsection{WAAM technology and CMT}

Wire Arc Additive Manufacturing (WAAM) is a process based on the deposit of wire beads with the use of [1] a heating source: the energy of a wire arc; a material tank in wire form; a displacement system. Various technologies can be used for WAAM, for instance, plasma arc (PAW) [2], arc welding with tungsten inert gas (TIG), gas metal arc welding (GMAW) with fuse wire and inert or active gas (MIG, MAG) [3] and Cold Metal Transfer (CMT) [4]. FRONIUS company developed in particular CMT as an enhanced system of GMAW [4]. Compared to the traditional GMAW, this process named cold welding can reduce heat input, which is an interesting point for additive manufacturing as the objective is to assemble beads for building a part; energy is therefore used to melt a part of the bead previously deposited. A too high energy provided to the bead can lead to material collapsing, thus makes it impossible to build the part. The process principle consists in a forward and backward movement of the wire. The bead is then deposited in

\footnotetext{
${ }^{1}$ Corresponding Author. thanh-hoang.vo@grenoble-inp.fr
} 
a series of drops. CMT process [4] has three main benefits compared to standard GMAW: a clean welding due to the quasi-absence of projections during process; a limited heat input thanks to the short-circuit; the arc stability as the arc length is set mechanically.

\subsection{Objectives and Scope}

The objectives of this research work is to propose a method to manufacture large parts with CMT technology. The study focuses on the strategy and manufacturing parameters with 5-axis trajectories. First of all, this article presents the state of the art about the main elements required for large parts manufacturing with WAAM, namely the various strategies and build parameters. Next section explains the manufacturing parameters that have an influence onto this type of parts. Several experimentations are then described in order to find which parameters need an adjustment. As a conclusion, major results are presented in terms of manufacturing parameters and filling strategy.

\section{State of the art}

\subsection{Manufacturing strategy}

The generation of trajectories is based on the part CAD model. A slicing step allows to get curves representing the manufacturing strategies. It is usually carried out through a series of parallel planes [5]. The analysis of the part geometry allows choosing the right trajectories [6], and two categories of areas arise: zones which wall thickness is below the width of a bead can be made with mono-beads, i.e. without filling. And zones which wall thickness is greater than the width of a bead stand out for needing a filling strategy. The result of these choices impacts the slicing as, for instance, the layer thickness can be different between a mono-bead and a multi-beads component. Three kinds of trajectories can thus be considered: 3 -axis, 5-axis and filling.

In order to manufacture large parts with WAAM, it is necessary to generate filling strategies by a deposit of beads side by side within the same layer. In that case, different scanning strategies can be considered: raster [7], zigzag [8], contour [9], spiral [10] (Figure 1). The raster strategy relies on the projection in one direction [7]. The zigzag approach combines the parallel lines separated in one continuous scan, which reduces the number of toolpath trajectories [8]. However, both approaches, raster and zigzag, give poor results because of the discretization applied onto any edge is not parallel to the tool path direction. As an alternative method, the contour strategy can solve this geometrical quality problem by following the geometrical trend of the part contours [9]. Lastly, the spiral trajectory is widely used in numerically controlled machining but does not fit for some specific geometrical models in AM process [10].

Therefore, WAAM configuration is a complex task as many parameters are involved and interdependent. Currently, few studies propose a generic method for selecting manufacturing parameters. Many researches focus their efforts on the influence of the manufacturing parameters onto the welding bead quality (geometry and material health). Dinovitzer et al. [11] applied Taguchi and ANOVA method for identifying the travel speed, the wire feed, the current as well as argon flow rates onto responses. This includes the shape, size, rugosity of beads, as well as the oxidation level, the fusion in depth and the microstructure. The travel speed and current have the biggest effect on responses. Increasing the travel speed or decreasing the current invoke a reduction of the fusion through the depth and a rugosity increase. Dahat et al. developed a method that allow to choose the current (Im) and travel speed (TS) for a given layer width (LW). These parameters help to remove less material during post-operations. 


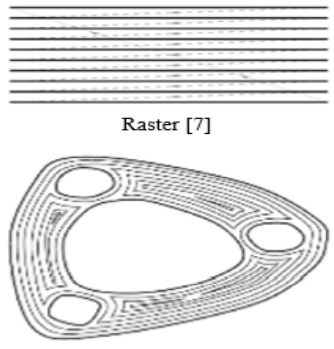

Contour [9]

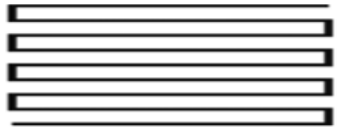

Zigzag [8]

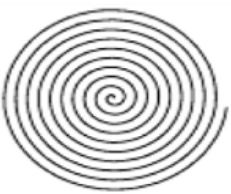

Spiral $[10]$

Figure 1. Filling strategies

\subsection{Conclusion}

Current researches show the CMT capacity for manufacturing large-dimension parts. This type of process is not easy to control as the parameters are different for each travel system and each material. Welding modes need indeed to adapt the energy input according to the material and wire diameter. Other studies that focused on mono-bead manufacturing have led to the selection of manufacturing parameters and strategies specific to thin parts, nonetheless, they have not been applied to large parts yet.

In this context, the research question is the following:

- What are the main difficulties for manufacturing large parts in WAAM-CMT? Especially:

- How to manufacture inclined parts, as well as geometrical singularities?

To answer these issues, the main challenge is to master the bead shape (width and height), as well as the heating temperature and the bead covering. Various experimentations in the next section show which parameters must be adjusted for obtaining a correct part. They are carried out with simple walls in aluminium, inclined walls and geometrical singularities. The model of a blade is used in a series of experimentations. By manufacturing a profile similar to a blade section, the aim is indeed to observe two issues: the manufacturing strategy and the choice of manufacturing parameters.

\section{Experimentations}

\subsection{Two manufacturing strategies}

In a first experience, the manufacturing strategy is tested on a large part. Figure 2 displays a part with a geometrical singularity, with two distinct zones in relation with the profile:

- Zone 1 which width is below the width of one bead

- Zone 2 which width is greater than the width of a bead

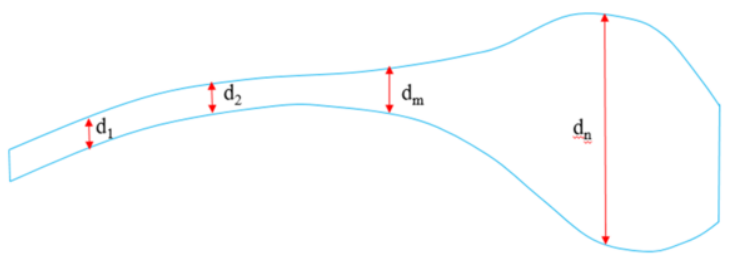

Figure 2. Example of part with a geometrical singularity 
Two manufacturing strategies are defined:

- Strategy 1: distinction of two areas in the profile: Zone 1 is produced in monobeads and Zone 2 by using zigzags (Figure 3).

- $\quad$ Strategy 2: use of zigzag all along the profile length (Figure 4).

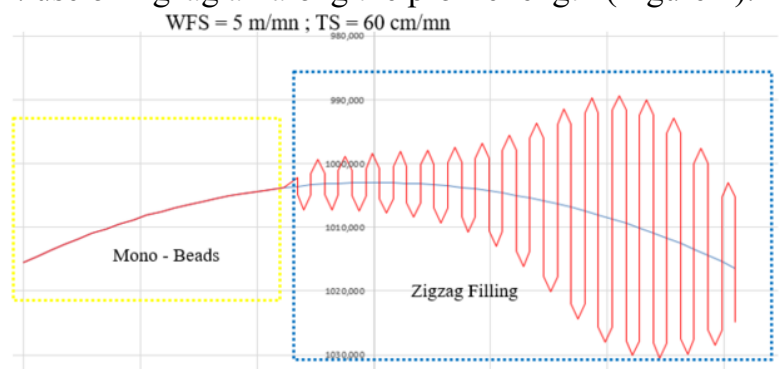

Figure 3. Strategy with mono-beads and zigzag filling

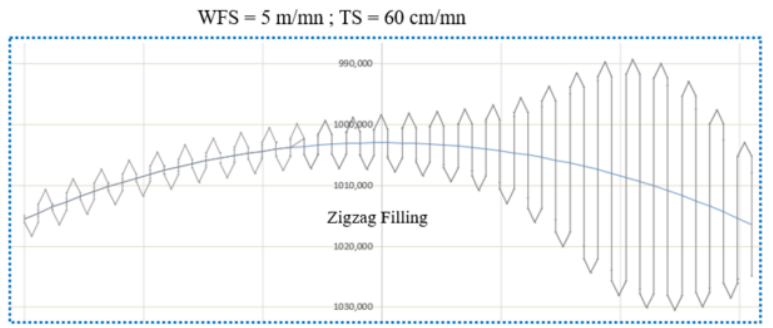

Figure 4. Strategy 2 with zigzag filling all along the part

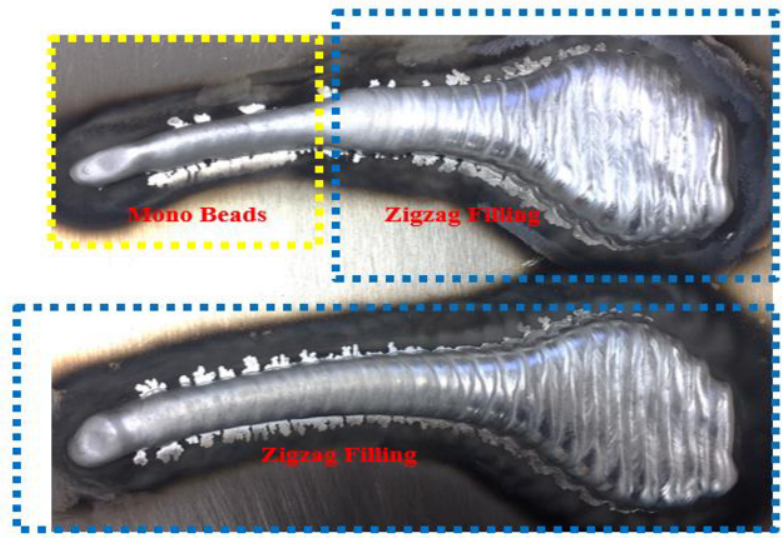

Figure 5. Results of both strategies with two layers

For both strategies, only two layers are built back and forth, in order to better observe the differences between both parts at this height (Figure 5). Concerning the part built with the first strategy, a gap greater than $2 \mathrm{~mm}$ is observed between the mono-bead zone and the filling zone. In addition, there is an irregularity in the width when passing from zone 1 to zone 2. Concerning the part built with strategy 2, the height is rather similar on the whole part, as of the second layer. Hence, from a surface and geometrical quality point of view, the second strategy is obviously more relevant than the first one. In order to study the average height of each layer, the same part is then built with more than two layers. 
3.2. Influence of manufacturing parameters onto the average height of the part A prototype with ten layers has been manufactured as shown in Figure 6.
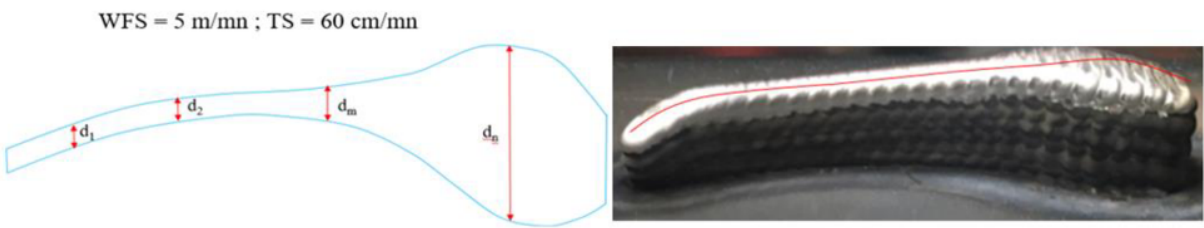

Figure 6. Prototype with ten layers

In this experimentation, the difference of height all along the part is outstanding. This height is lower at both sides of the part because of the edges effect. The height of the part is different according to the variation of the bead length $(\mathrm{dn})$. The area where the bead is the longest is the thickest. This problem can be explained as follows: when the zigzag length (d) is greater, the back and forth manufacturing time takes longer. The required cooling time is therefore more important, and the temperature of spreading is colder, which entails the manufacturing of more narrow and higher beads.

Another prototype of ten layers has been manufactured by modifying the torch speed (TS) with regard to the bead length, so as to obtain an identical height (of 4,5 $\mathrm{mm}$ ) all over the part (Figure 7).

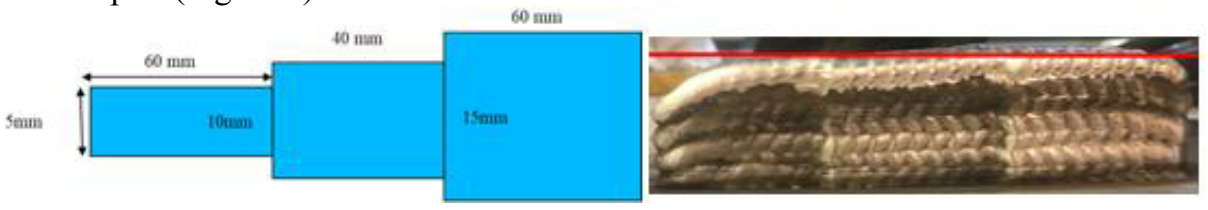

Figure 7. Another prototype with three different beads length

For each bead length, large walls have been built with two different torch speeds, 5 $\mathrm{cm} / \mathrm{s}$ et $10 \mathrm{~cm} / \mathrm{s}$. The average height of each prototype was measured. It was considered that the relationship between the torch speeds and the average height is a superlative equation (Figure 8) which allows to calculate TS and get an average height of 4,5 $\mathrm{mm}$.

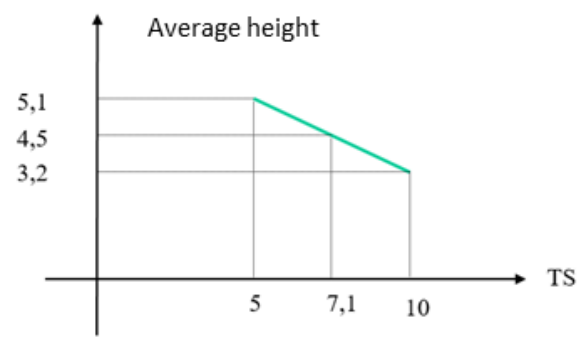

Figure 8. Relation between TS and average height

Usually, the height of the part is rather stable. An average height of 4,6mm was obtained unlike the theoretical 4,5 $\mathrm{mm}$. In addition, the same issues arose at the edges of the part and within the areas where the bead length changes. As for the manufacturing strategy, we recommend to use the zigzag filling strategy, regardless of the part width. For the manufacturing parameters, the existing model for parameters selection suits for a part with mono-beads but not for a large part because it leads to a height problem. Consequently, a new model needs to be developed in order to select appropriate parameters. 


\section{Conclusion and perspectives}

The challenge of manufacturing a large part in WAAM lies in the fact that the part must have a constant height whatever its length and width, to be able to consider it as of good quality. Moreover, for a large part with a geometrical singularity, we strove to identify the best filling strategy as well as the manufacturing parameters that have an influence onto the part quality. In conclusion:

- A zigzag filling strategy works fine.

- A correctly determined value of the incremented in $\mathrm{z}$ leads to a good part geometry, i.e. the same as the CAD model one.

- To build a good-quality prototype, the torch speed (TS) must vary according to the bead length.

This research study provides key elements for manufacturing multi-beads large parts with WAAM. Although its results do not help to determine the manufacturing parameters yet, the following perspectives can be suggested:

- Improve the method proposed by Quérard [6] for large parts with WAAM.

- Propose a model to calculate the torch speed (TS) based on the bead length.

\section{References}

[1] W. S. Filomeno Martina, Wire + arc additive vs. from solid: a cost comparison, Welding Engineering and Laser Processing Centre, Cranfield University, 2015.

[2] D. Ding, Z. Pan, D. Cuiuri, and H. Li, Wire-feed additive manufacturing of metal components: technologies, developments and future interests, Int $J A d v$ ManufTechnol, 2014.

[3] D. Ding, Z. Pan, D. Cuiuri, and H. Li, A multi-bead overlapping model for robotic wire and arc additive manufacturing (WAAM), Robotics and Computer-Integrated Manufacturing, vol. 31, pp. 101-110, 2015.

[4] Fronius, Cold Metal Transfer: The technology, CMT technology, 2014.

[5] J. S. Panchagnula and S. Simhambhatla, Inclined slicing and weld-deposition for additive manufacturing of metallic objects with large overhangs using higher order kinematics, Virtual and Physical Prototyping, vol. 11, no. 2, pp. 99-108, 2016.

[6] Querard, Vincent. Réalisation de pièces aéronautiques de grandes dimensions par fabrication additive WAAM. Diss. 2019.

[7] Dunlavey, M.R., Efficient polygon-filling algorithms for raster displays. ACM Transactions on Graphics (Tog), 2(4), pp.264-273, 1983.

[8] Park, S.C. and Choi, B.K., Tool-path planning for direction-parallel area milling. Computer-Aided Design, 32(1), pp.17-25, 2000.

[9] Farouki, R.T., Koenig, T., Tarabanis, K.A., Korein, J.U. and Batchelder, J.S., Path planning with offset curves for layered fabrication processes. Journal of Manufacturing Systems, 14(5), pp.355-368, 1995.

[10] Li, H., Dong, Z. and Vickers, G.W., Optimal toolpath pattern identification for single island, sculptured part rough machining using fuzzy pattern analysis. Computer-Aided Design, 26(11), pp.787-795, 1994.

[11] Dinovitzer, M., Chen, X., Laliberte, J., Huang, X. and Frei, H., Effect of wire and arc additive manufacturing (WAAM) process parameters on bead geometry and microstructure. Additive Manufacturing, 26, pp.138-146, 2019. 